\title{
A CONSTRUCTION OF $\mathrm{N}=2$ AND CENTERLESS $\mathrm{N}=4$ SUPERCONFORMAL FIELDS VIA AFFINE SUPERALGEBRAS
}

\author{
Minoru Wakimoto \\ Graduate School of Mathematics, Kyushu University, Fukuoka 812-81, \\ Japan
}

\begin{abstract}
In this note we give a new construction of the $\mathrm{N}=2$ superconformal algebra using currents of the affine superalgebra $\widehat{s l}(2 \mid 1)$ and free bosonic fields, and also the $\mathrm{N}=4$ superconformal algebra without central charge in terms of currents of $\widehat{s l}(2 \mid 2)$ and free bosonic fields.
\end{abstract}

\section{IntroduCtion}

There seem to be several ways to construct the $\mathrm{N}=2$ superconformal algebra via affine Lie algebras or affine superalgebras by tensoring with some fermionic or bosonic fields. In this note, first we give a simple method to construct it from the affine superalgebra $\widehat{s l}(2 \mid 1)$ with the help of two free bosonic fields and their dual fields. This construction is achieved by a calculus of the inner product and the structure of root systems. A very particular feature is that the similar construction does not work for $\widehat{s l}(3, \mathbb{C})$ to get a finite-rank algebra, but only gives an algebra of infinite rank just like the famous Zamolodchikov's W-algebra. For $\widehat{s l}(2 \mid 1)$, however, the operator product is well-behaved and gives a family of finite fields, namely the $\mathrm{N}=2$ superconformal algebra. We see in this construction that the existence of odd simple roots with zero square length play a very important role. So our calculus is not described only by the terminology of root systems, but the inner product is concerned.

Since the construction works so well for the simplest superalgebra $\widehat{s l}(2 \mid 1)$, one will naturally ask how to extend this method to get higher rank superconformal algebras. It is not clear, however, whether superconformal algebras with fields of negative conformal weights are expected to be obtained in such a way associated to affine superalgebras. It may be expected for physical superconformal algebras which, by definition, do not admit fields of non-positive conformal weights and are 
completely classified by [5]. But actually the difficulty arises soon in the higher rank case to get a family of finite numbers of fields closed under the operator product. In this note, we propose a way to construct the $\mathrm{N}=4$ superconformal algebra, although with zero central charge, associated to the affine superalgebra $\widehat{A}(1,1)$, namely the affinization of the finite-dimensional simple superalgebra $s l(2 \mid 2)$ divided by its center. We find a family of finite fields which are almost closed under the operator products, and an algebra of finite rank is obtained by factorizing this vertex algebra by an ideal with a single generator. This procedure of factorization, however, enforces us to retreat to the zero central charge.

In our construction, the $\mathrm{N}=2$ superconformal algebra acts on the space of tensor procduct of an irreducible highest weight $\widehat{s l}(2 \mid 1)$-module and the Clifford module, and also similar for the $\mathrm{N}=4$ superconformal algebra. We find its invariant subspaces by using commutants. The analysis of their irreducibility and characters remains a problem.

\section{SOME FORMUlas FOR OPERATOR PRODUCTS}

Throughout this paper we make use of the physicists' convention of the operator product

$$
a(z) b(w)=\sum_{j=1}^{n} \frac{c_{j}(w)}{(z-w)^{j}}
$$

to denote

$$
[a(z), b(w)]=\sum_{j=1}^{n} c_{j}(w) \frac{\partial_{w}^{j-1}}{(j-1) !} \delta(z-w) .
$$

Let $\mathfrak{g}$ be a finite-dimensional simple superalgebra with an even nondegenerate super-invariant super-symmetric bilinear form $(\mid)$, and $\hat{\mathfrak{g}}$ be its affinization. Here "even" means, by definition, that the even part of $\mathfrak{g}$ is orthogonal to the odd part with respect to ( $\mid$ ) (cf. [2]). Let $\Delta$ be the set of all roots of $\mathfrak{g}$ with respect to its Cartan subalgebra $\mathfrak{h}$, and $\Delta^{+}$(resp. $\Delta^{-}$) the subset of $\Delta$ consisting of all positive (resp. negative) roots. Also let $\Delta_{\text {even }}$ (resp. $\Delta_{\text {odd }}$ ) denote the set of all even (resp. odd) roots. As usual, for a root $\alpha, X_{\alpha}$ is an element from the corresponding root space $\mathfrak{g}_{\alpha}$, and $H_{\alpha}$ is the element in $\mathfrak{h}$ satisfying $\alpha(h)=\left(H_{\alpha} \mid h\right)$ for all $h \in \mathfrak{h}$.

For each $\alpha \in \Delta^{+}$, we introduce two fields of conformal weight $\frac{1}{2}$ :

$$
\psi_{\alpha}(z)=\sum_{n \in \mathbb{Z}} \psi_{\alpha}(n) z^{-n-\frac{1}{2}}=\sum_{n \in \frac{1}{2}+\mathbb{Z}} \psi_{\alpha}\left(n+\frac{1}{2}\right) z^{-n-1}
$$


and

$$
\psi^{\alpha}(z)=\sum_{n \in \mathbb{Z}} \psi^{\alpha}(n) z^{-n-\frac{1}{2}}=\sum_{n \in \frac{1}{2}+\mathbb{Z}} \psi^{\alpha}\left(n+\frac{1}{2}\right) z^{-n-1} .
$$

These fields are fermionic or bosonic according as the associated root $\alpha$ is even or odd, and assumed to satisfy the (anti-)commutation relations

$$
\left[\psi^{\alpha}(m), \psi_{\beta}(n)\right]=(-1)^{p(\alpha)}\left[\psi_{\beta}(n), \psi^{\alpha}(m)\right]=\delta_{\alpha, \beta} \delta_{m+n, 0},
$$

where $p(\alpha)$ denotes the parity of a root $\alpha$, namely is equal to 0 if $\alpha$ is even and 1 if $\alpha$ is odd. We extend $\psi^{\alpha}$ and $\psi_{\alpha}$ to negative roots by $\psi_{-\alpha}(z)=(-1)^{p(\alpha)} \psi^{\alpha}(z)$ and $\psi^{-\alpha}(z)=\psi_{\alpha}(z)$ for each positive root $\alpha$. These relations are simply written as

$$
\psi_{\alpha}(z)=\epsilon(\alpha) \psi^{-\alpha}(z)
$$

and

$$
\psi^{\alpha}(z)=(-1)^{p(\alpha)} \epsilon(\alpha) \psi_{-\alpha}(z)
$$

for all roots $\alpha$, where

$$
\epsilon(\alpha):= \begin{cases}1 & \text { if } \alpha \text { is positive } \\ (-1)^{p(\alpha)} & \text { if } \alpha \text { is negative. }\end{cases}
$$

For a positive root $\alpha$, the operators $\psi^{\alpha}(m)$ with $m<0$ (resp. $m \geq 0$ ) and $\psi_{\alpha}(n)$ with $n \leq 0$ (resp. $n>0$ ) are assumed to be creation (resp. annihilation) operators. Using the inner product

$$
\left(\psi^{\alpha} \mid \psi^{\beta}\right)=\left(\psi_{\alpha} \mid \psi_{\beta}\right):=\epsilon(\alpha) \delta_{\alpha+\beta, 0}
$$

or equivalently

$$
\left(\psi^{\alpha} \mid \psi_{\beta}\right)=(-1)^{p(\alpha)}\left(\psi_{\alpha} \mid \psi^{\beta}\right):=\delta_{\alpha, \beta},
$$

the operator products of these fields are simply written as the following formula:

$$
\varphi(z) \psi(w)=\frac{(\varphi \mid \psi)}{z-w},
$$

where $\varphi(z)$ and $\psi(z)$ are elements from $\psi^{\alpha}(z)$ 's and $\psi_{\alpha}(z)$ 's.

For each element $X \in \mathfrak{g}$, the field

$$
X(z):=\sum_{n \in Z} X(n) z^{-n-1}
$$

is called a current, where $X(n)$ 's, $n \in \mathbb{Z}$, are elements in the affinization $\widehat{\mathfrak{g}}$ of $\mathfrak{g}$, satisfying the (anti-)commutation relation

$$
[X(m), Y(n)]=[X, Y](m+n)+m(X \mid Y) \delta_{m+n, 0} K
$$


where $K$ is the canonical central element in $\widehat{\mathfrak{g}}$.

In terms of the operator product, the formula (1.6a) is written as

$$
X(z) Y(w)=\frac{(X \mid Y) K}{(z-w)^{2}}+\frac{[X, Y](w)}{z-w}
$$

In this note, $a(z), b(z), c(z), \cdots, X(z), Y(z), \cdots$ always stand for currents, and $\psi(z), \varphi(z), \cdots$ free fields with product (1.5), and $p()$ denotes the parity of a field. We note that

$$
p\left(\psi^{\alpha}\right)=p\left(\psi_{\alpha}\right)=p(\alpha)+1 \quad \bmod 2 \mathbb{Z} .
$$

A very important tool in our operator calculus is the Wick theorem in particular for non-commutative fields, a clear explanation on which is given, e.g., in Section 3.3 of 1 . Here is a list of formulas obtained from the non-commutative Wick theorem, which will be used in this paper.

$$
\begin{aligned}
& : a(z) b(z):: c(w):=\frac{-([a, b] \mid c) K}{(z-w)^{3}}+(b \mid c) K\left\{\frac{a(w)}{(z-w)^{2}}+\frac{\partial a(w)}{z-w}\right\} \\
& +(-1)^{p(b) p(c)}(a \mid c) K\left\{\frac{b(w)}{(z-w)^{2}}+\frac{\partial b(w)}{z-w}\right\}+(-1)^{p(a) p(b)} \frac{[b,[a, c]](w)}{\left(z-\left(\Psi . \delta^{2} a\right)\right.} \\
& +\frac{1}{z-w}\left\{: a(w)[b, c](w):+(-1)^{p(a) p(b)}: b(w)[a, c](w):\right\}, \\
& : a(z):: b(w) c(w):=\frac{(a \mid[b, c]) K}{(z-w)^{3}} \\
& +\frac{1}{(z-w)^{2}}\left\{(a \mid b) K \cdot c(w)+(-1)^{p(a) p(b)}(a \mid c) K \cdot b(w)+[[a, b], c](w)\right\} \\
& +\frac{1}{z-w}\left\{:[a, b](w) c(w):+(-1)^{p(a) p(b)}: b(w)[a, c](w):\right\}, \\
& (-1)^{p(\varphi) p(Y)}: X(z) \varphi(z):: Y(w) \psi(w):=(X \mid Y)(\varphi \mid \psi) \frac{K}{(z-w)^{3}} \\
& +(\varphi \mid \psi) \frac{[X, Y](w)}{(z-w)^{2}}+(X \mid Y) K\left\{\frac{: \varphi(w) \psi(w):}{(z-w)^{2}}+\frac{: \partial \varphi(w) \psi(w):}{z-w}\right\}_{9)} \\
& +\frac{1}{z-w}\{[x, y](w): \varphi(w) \psi(w):+(\varphi \mid \psi): X(w) Y(w):\},
\end{aligned}
$$




$$
\begin{aligned}
& (-1)^{p(X) p(\varphi)}: X(z) \varphi(z):: Y(w) Z(w): \\
& =([X, Y] \mid Z) K\left\{\frac{\varphi(w)}{(z-w)^{3}}+\frac{\partial \varphi(w)}{(z-w)^{2}}+\frac{\frac{1}{2} \partial^{2} \varphi(w)}{z-w}\right\} \\
& +(X \mid Y) K\left\{\frac{\varphi(w) Z(w)}{(z-w)^{2}}+\frac{\partial \varphi(w) Z(w)}{z-w}\right\} \\
& +(-1)^{p(X) p(Y)}(X \mid Z) K\left\{\frac{\varphi(w) Y(w)}{(z-w)^{2}}+\frac{\partial \varphi(w) Y(w)}{z-w}\right\} \\
& +\frac{\varphi(w)[[X, Y], Z](w)}{(z-w)^{2}}+\frac{\partial \varphi(w)[[X, Y], Z](w)}{z-w} \\
& +\frac{1}{z-w} \varphi(w):[X, Y](w) Z(w):+\frac{(-1)^{p(X) p(Y)}}{z-w} \varphi(w): Y(w)[X, Z](w):, \\
& (-1)^{p(Y)\left(p\left(\varphi_{1}\right)+p\left(\varphi_{2}\right)\right)}: X(z) \varphi_{1}(z) \varphi_{2}(z):: Y(w) \psi(w): \\
& =(X \mid Y) K\left(\varphi_{2} \mid \psi\right)\left\{\frac{\varphi_{1}(w)}{(z-w)^{3}}+\frac{2 \partial \varphi_{1}(w)}{(z-w)^{2}}+\frac{\frac{3}{2} \partial^{2} \varphi_{1}(w)}{z-w}\right\} \\
& +(-1)^{p\left(\varphi_{2}\right) p(\psi)}(X \mid Y) K\left(\varphi_{1} \mid \psi\right)\left\{\frac{\varphi_{2}(w)}{(z-w)^{3}}+\frac{2 \partial \varphi_{2}(w)}{(z-w)^{2}}+\frac{\frac{3}{2} \partial^{2} \varphi_{2}(w)}{z-w}\right\} \\
& +(X \mid Y) K\left\{\frac{: \varphi_{1}(w) \varphi_{2}(w) \psi(w):}{(z-w)^{2}}+\frac{: \partial\left(\varphi_{1}(w) \varphi_{2}(w)\right) \psi(w):}{z-w}\right\} \\
& +\left(\varphi_{2} \mid \psi\right)\left\{\frac{[X, Y](w) \varphi_{1}(w)}{(z-w)^{2}}+\frac{[X, Y](w) \partial \varphi_{1}(w)}{z-w}+\frac{: X(w) Y(w): \varphi_{1}(w)}{z-w}\right\} \\
& +(-1)^{p\left(\varphi_{2}\right) p(\psi)}\left(\varphi_{1} \mid \psi\right)\left\{\frac{[X, Y](w) \varphi_{2}(w)}{(z-w)^{2}}+\frac{[X, Y](w) \partial \varphi_{2}(w)}{z-w}+\frac{: X(w) Y(w): \varphi_{2}(w)}{z-w}\right\} \\
& +\frac{[X, Y](w): \varphi_{1}(w) \varphi_{2}(w) \psi(w):}{z-w} .
\end{aligned}
$$

In particular from (1.8) and (1.9) one deduces the following:

Lemma 1.1. Let $\alpha, \beta \in \Delta$ such that $\alpha+\beta \neq 0$, then

$: X_{\alpha}(z) \psi^{\alpha}(z):: X_{\beta}(w) \psi^{\beta}(w):=\frac{(-1)^{(p(\alpha)+1) p(\beta)}}{z-w}\left[X_{\alpha}, X_{\beta}\right](w): \psi^{\alpha}(w) \psi^{\beta}(w):$.

Lemma 1.2. Let $H \in \mathfrak{h}$ and $X_{\alpha} \in \mathfrak{g}_{\alpha}$ and $X_{-\alpha} \in \mathfrak{g}_{-\alpha}$ such that $\left[X_{\alpha}, X_{-\alpha}\right]=H_{\alpha} ;$ then 
1)

$$
H(z): X_{\alpha}(w) X_{-\alpha}(w):=\frac{\left(H_{\alpha} \mid H\right) K}{(z-w)^{3}}+\alpha(H) \frac{H_{\alpha}(w)}{(z-w)^{2}},
$$

$: X_{\alpha}(z) X_{-\alpha}(z): H(w)=\frac{-\left(H_{\alpha} \mid H\right) K}{(z-w)^{3}}+\alpha(H)\left\{\frac{H_{\alpha}(w)}{(z-w)^{2}}+\frac{\partial H_{\alpha}(w)}{z-w}\right\}$,

$: X_{\alpha}(z) \psi^{\alpha}(z):: X_{-\alpha}(w) \psi^{-\alpha}(w)$ :

$=\epsilon(\alpha) \frac{\left(X_{\alpha} \mid X_{-\alpha}\right) K}{(z-w)^{3}}+\frac{\epsilon(\alpha)}{(z-w)^{2}}\left\{\left(X_{\alpha} \mid X_{-\alpha}\right) K: \psi^{\alpha}(w) \psi_{\alpha}(w):+H_{\alpha}(w)\right\}$

$+\frac{\epsilon(\alpha)}{z-w}\left\{: X_{\alpha}(w) X_{-\alpha}(w):+H_{\alpha}(w): \psi^{\alpha}(w) \psi_{\alpha}(w):\right.$

$\left.+\left(X_{\alpha} \mid X_{-\alpha}\right) K: \partial \psi^{\alpha}(w) \psi_{\alpha}(w):\right\}$.

\section{The $\mathrm{N}=2$ Superconformal algebra Associated to $\widehat{s l}(2 \mid 1)$}

In this section we start our discussion under a general situation, where $\mathfrak{g}$ is a simple Lie superalgebra with an even non-degenerate superinvariant super-symmetric bilinear form ( $\mid$ ). One can choose $H_{\alpha}$ and $X_{\alpha}$ such that

$$
\begin{gathered}
\left(X_{\alpha} \mid X_{-\alpha}\right)=(-1)^{p(\alpha)} \epsilon(\alpha), \\
H_{\alpha}=(-1)^{p(\alpha)} \epsilon(\alpha) \alpha .
\end{gathered}
$$

Note that

$$
H_{-\alpha}=-(-1)^{p(\alpha)} H_{\alpha}
$$

Given a pair $(\alpha, \beta)$ of roots such that

(the linear span of $\alpha$ and $\beta) \cap \Delta=\{ \pm \alpha, \pm \beta, \pm(\alpha+\beta)\}$,

we introduce the following fields:

$$
\begin{aligned}
G_{+}(z) & :=X_{\alpha}(z) \psi^{\alpha}(z)+X_{-\beta}(z) \psi^{-\beta}(z), \\
G_{-}(z) & :=X_{\beta}(z) \psi^{\beta}(z)+X_{-\alpha}(z) \psi^{-\alpha}(z), \\
A(z) & :=K\left\{(-1)^{p(\alpha)} \epsilon(\alpha): \psi^{\alpha}(z) \psi_{\alpha}(z):-(-1)^{p(\beta)} \epsilon(\beta): \psi^{\beta}(z) \psi_{\beta}(z):\right\} \\
& +H_{\alpha}(z)-H_{\beta}(z), \\
B(z) & :=K\left\{(-1)^{p(\alpha)} \epsilon(\alpha): \partial \psi^{\alpha}(z) \psi_{\alpha}(z):-(-1)^{p(\beta)} \epsilon(\beta): \psi^{\beta}(z) \partial \psi_{\beta}(z):\right\} \\
& +: X_{\alpha}(z) X_{-\alpha}(z):+: X_{\beta}(z) X_{-\beta}(z):-\partial H_{\beta}(z) \\
& 6
\end{aligned}
$$




$$
\begin{aligned}
& +H_{\alpha}(z): \psi^{\alpha}(z) \psi_{\alpha}(z):+H_{\beta}(z): \psi^{\beta}(z) \psi_{\beta}(z): \\
& +(-1)^{p(\alpha) p(\beta)} c_{\alpha, \beta}\left\{(-1)^{p(\beta)}: X_{\alpha+\beta}(z) \psi^{\alpha}(z) \psi^{\beta}(z):\right. \\
& \left.-(-1)^{p(\alpha)} \epsilon(\alpha) \epsilon(\beta): X_{-\alpha-\beta}(z) \psi_{\alpha}(z) \psi_{\beta}(z):\right\} .
\end{aligned}
$$

In the actual calculation of operator products of fields, one needs the following relations among structure constants, which are deduced from the Jacobi identity:

$$
\begin{aligned}
c_{-\alpha,-\beta} & =-(-1)^{p(\alpha+\beta)} c_{\alpha, \beta}, \\
c_{\alpha,-\alpha-\beta} & =-(-1)^{(p(\alpha)+1) p(\beta)} \epsilon(\alpha) c_{\alpha, \beta}, \\
c_{\beta,-\alpha-\beta} & =(-1)^{p(\alpha)} \epsilon(\beta) c_{\alpha, \beta}, \\
c_{-\alpha, \alpha+\beta} & =(-1)^{p(\alpha) p(\beta)} \epsilon(\alpha) c_{\alpha, \beta}, \\
c_{-\beta, \alpha+\beta} & =-\epsilon(\beta) c_{\alpha, \beta} .
\end{aligned}
$$

Then, by Lemmas 1.1 and 1.2, one easily has the following:

\section{Lemma 2.1.}

1)

2)

$$
G_{+}(z) G_{+}(w)=G_{-}(z) G_{-}(w)=0
$$

$$
\begin{aligned}
& G_{+}(z) G_{-}(w) \\
& =\left\{(-1)^{p(\alpha)} \epsilon(\alpha)+(-1)^{p(\beta)} \epsilon(\beta)\right\} \frac{K}{(z-w)^{3}}+\frac{A(w)}{(z-w)^{2}}+\frac{B(w)}{z-w} .
\end{aligned}
$$

And the following is shown by a simple calculation using (1.8),(1.9) and (1.10):

$$
A(z): X_{\alpha}(w) \psi^{\alpha}(w):
$$$$
=\left\{\epsilon(\alpha) K+(-1)^{p(\alpha)} \epsilon(\alpha)(\alpha \mid \alpha)-(-1)^{p(\beta)} \epsilon(\beta)(\alpha \mid \beta)\right\} \frac{X_{\alpha}(w) \psi^{\alpha}(w)}{z-w},
$$

$$
A(z): X_{-\beta}(w) \psi^{-\beta}(w):
$$

$$
=\left\{\epsilon(\beta) K-(-1)^{p(\alpha)} \epsilon(\alpha)(\alpha \mid \beta)+(-1)^{p(\beta)} \epsilon(\beta)(\beta \mid \beta)\right\} \frac{X_{-\beta}(w) \psi^{-\beta}(w)}{z-w},
$$

$$
A(z): X_{\beta}(w) \psi^{\beta}(w):
$$

$$
=\left\{-\epsilon(\beta) K+(-1)^{p(\alpha)} \epsilon(\alpha)(\alpha \mid \beta)-(-1)^{p(\beta)} \epsilon(\beta)(\beta \mid \beta)\right\} \frac{X_{\beta}(w) \psi^{\beta}(w)}{z-w},
$$




$$
A(z): X_{-\alpha}(w) \psi^{-\alpha}(w):
$$

$$
=\left\{-\epsilon(\alpha) K-(-1)^{p(\alpha)} \epsilon(\alpha)(\alpha \mid \alpha)+(-1)^{p(\beta)} \epsilon(\beta)(\alpha \mid \beta)\right\} \frac{X_{-\alpha}(w) \psi^{-\alpha}(w)}{z-w} \text {. }
$$

From these, one has the following:

Lemma 2.2. Assume that $(\alpha \mid \alpha)=(\beta \mid \beta)=0,(\alpha \mid \beta)=1$, and $\epsilon(\alpha)=$ $\epsilon(\beta)=1$. Then

1)

2)

$$
A(z) G_{+}(w)=\frac{K+1}{z-w} G_{+}(w)
$$

$$
A(z) G_{-}(w)=\frac{-(K+1)}{z-w} G_{-}(w) .
$$

We now proceed to compute the operator product of $B(z)$ with $G_{ \pm}(z)$. After a long and tiresome calculation using (1.9), (1.10) and (1.11), one arrives at the following:

$$
\begin{aligned}
& \text { 7a) } B(z): X_{\alpha}(w) \psi^{\alpha}(w): \\
& =\epsilon(\alpha)\{K+(\alpha \mid \alpha)\}\left\{\frac{X_{\alpha}(w) \psi^{\alpha}(w)}{(z-w)^{2}}+\frac{\partial\left(X_{\alpha}(w) \psi^{\alpha}(w)\right)}{z-w}\right\} \\
& +(-1)^{(p(\alpha)+1) p(\beta)} \epsilon(\beta)\left\{\frac{X_{\alpha}(w) \psi^{\alpha}(w)}{(z-w)^{2}}+\frac{\partial X_{\alpha}(w) \psi^{\alpha}(w)}{z-w}\right\} \\
& +(-1)^{p(\beta)} \epsilon(\beta)(\alpha \mid \beta) \frac{X_{\alpha}(w) \psi^{\alpha}(w)}{(z-w)^{2}} \\
& +(-1)^{p(\alpha)(p(\beta)+1)}\left\{\frac{X_{-\beta}(w) \psi^{-\beta}(w)}{(z-w)^{2}}+\frac{\partial\left(X_{-\beta}(w) \psi^{-\beta}(w)\right)}{z-w}\right\} \\
& +(-1)^{p(\alpha)+p(\beta)} \epsilon(\alpha) \epsilon(\beta) \frac{c_{\alpha, \beta}}{z-w}: X_{\alpha}(w) X_{-\alpha-\beta}(w) \psi_{\beta}(w): \\
& -(-1)^{p(\alpha)+p(\beta)} \frac{c_{\alpha, \beta}}{z-w}: X_{\alpha+\beta}(w) X_{-\beta}(w) \psi^{\alpha}(w): \\
& +(-1)^{p(\alpha)} \epsilon(\alpha) \frac{(\alpha \mid \alpha)}{z-w}: X_{\alpha}(w) \psi^{\alpha}(w) \psi_{\alpha}(w) \psi^{\alpha}(w): \\
& +(-1)^{p(\beta)} \epsilon(\beta) \frac{(\alpha \mid \beta)}{z-w}: X_{\alpha}(w) \psi^{\alpha}(w) \psi^{\beta}(w) \psi_{\beta}(w): \\
& +(-1)^{p(\alpha) p(\beta)} \frac{\epsilon(\beta)}{z-w}: X_{-\beta}(w) \psi_{\beta}(w) \psi_{\alpha}(w) \psi^{\alpha}(w): \\
& =\epsilon(\beta)\{K+(\beta \mid \beta)\}\left\{\frac{X_{-\beta}(w) \psi^{-\beta}(w)}{(z-w)^{2}}+\frac{\partial\left(X_{-\beta}(w) \psi^{-\beta}(w)\right)}{z-w}\right\} \\
& B(z): X-\beta(w) \psi^{-\beta}(w):
\end{aligned}
$$




$$
\begin{aligned}
& +(-1)^{(p(\alpha)+1) p(\beta)}\left\{\frac{X_{\alpha}(w) \psi^{\alpha}(w)}{(z-w)^{2}}+\frac{X_{\alpha}(w) \partial \psi^{\alpha}(w)}{z-w}\right\} \\
& +\epsilon(\beta)(\beta \mid \beta)\left\{1-(-1)^{p(\beta)}\right\} \frac{X_{-\beta}(w) \psi^{-\beta}(w)}{(z-w)^{2}} \\
& -(-1)^{p(\alpha)+p(\beta)} \epsilon(\beta) \frac{c_{\alpha, \beta}}{z-w}: X_{\alpha}(w) X_{-\alpha-\beta}(w) \psi_{\beta}(w): \\
& +\epsilon(\beta) \frac{c_{\alpha, \beta}}{z-w}: X_{\alpha+\beta}(w) X_{-\beta}(w) \psi^{\alpha}(w): \\
& -(-1)^{p(\beta)} \frac{(\beta \mid \beta)}{z-w}: X_{-\beta}(w) \psi^{\beta}(w) \psi_{\beta}(w) \psi_{\beta}(w): \\
& -(-1)^{p(\alpha)} \epsilon(\alpha) \epsilon(\beta) \frac{(\alpha \mid \beta)}{z-w}: X_{-\beta}(w) \psi_{\beta}(w) \psi^{\alpha}(w) \psi_{\alpha}(w): \\
& +(-1)^{(p(\alpha)+1) p(\beta)} \frac{1}{z-w}: X_{\alpha}(w) \psi^{\alpha}(w) \psi^{\beta}(w) \psi_{\beta}(w):
\end{aligned}
$$

$$
\begin{aligned}
& =\epsilon(\beta)\{K+(\beta \mid \beta)\}\left\{\frac{X_{\beta}(w) \psi^{\beta}(w)}{(z-w)^{2}}+\frac{\partial\left(X_{\beta}(w) \psi^{\beta}(w)\right)}{z-w}\right\} \\
& +(-1)^{p(\alpha)(p(\beta)+1)} \epsilon(\alpha)\left\{\frac{X_{\beta}(w) \psi^{\beta}(w)}{(z-w)^{2}}+\frac{\partial X_{\beta}(w) \psi^{\beta}(w)}{z-w}\right\} \\
& +\epsilon(\beta)\left\{K+(-1)^{p(\beta)}(\beta \mid \beta)\right\} \frac{X_{\beta}(w) \psi^{\beta}(w)}{(z-w)^{2}} \\
& +(-1)^{(p(\alpha)+1) p(\beta)}\left\{\frac{X_{-\alpha}(w) \psi^{-\alpha}(w)}{(z-w)^{2}}+\frac{\partial\left(X_{-\alpha}(w) \psi^{-\alpha}(w)\right)}{z-w}\right\} \\
& +(-1)^{(p(\alpha)+1)(p(\beta)+1)} \epsilon(\alpha) \epsilon(\beta) \frac{c_{\alpha, \beta}}{z-w}: X_{\beta}(w) X_{-\alpha-\beta}(w) \psi_{\alpha}(w): \\
& \left.-(-1)^{(p(\alpha)+1)(p(\beta)+1)} \frac{c_{\alpha, \beta}: X_{\alpha+\beta}(w) X_{-\alpha}(w) \psi^{\beta}(w):}{z-w}, \psi^{\alpha}\right) \\
& +(-1)^{p(\alpha)} \epsilon(\alpha) \frac{(\alpha \mid \beta)}{z-w}: X_{\beta}(w) \psi^{\beta}(w) \psi^{\alpha}(w) \psi_{\alpha}(w): \\
& +(-1)^{p(\beta)} \epsilon(\beta) \frac{(\beta \mid \beta)}{z-w}: X_{\beta}(w) \psi^{\beta}(w) \psi_{\beta}(w) \psi^{\beta}(w): \\
& -(-1)^{(p(\alpha)+1) p(\beta)} \frac{\epsilon(\beta)}{z-w}: X_{-\alpha}(w) \psi_{\alpha}(w) \psi^{\beta}(w) \psi_{\beta}(w): \\
& B(z): X \psi_{-\alpha}(w) \psi^{-\alpha}(w): \\
& =\epsilon(\alpha)\{K+(\alpha \mid \alpha)\}\left\{\frac{X_{-\alpha}(w) \psi^{-\alpha}(w)}{(z-w)^{2}}+\frac{\partial\left(X_{-\alpha}(w) \psi^{-\alpha}(w)\right)}{z-w}\right\}
\end{aligned}
$$




$$
\begin{aligned}
& +(-1)^{p(\alpha)(p(\beta)+1)}\left\{\frac{X_{\beta}(w) \psi^{\beta}(w)}{(z-w)^{2}}+\frac{X_{\beta}(w) \partial \psi^{\beta}(w)}{z-w}\right\} \\
& +\left\{\epsilon(\alpha)(K+(\alpha \mid \alpha))-(-1)^{p(\beta)} \epsilon(\beta)(\alpha \mid \beta)\right\} \frac{X_{-\alpha}(w) \psi^{-\alpha}(w)}{(z-w)^{2}} \\
& -(-1)^{(p(\alpha)+1)(p(\beta)+1)} \epsilon(\alpha) \frac{c_{\alpha, \beta}}{z-w}: X_{\beta}(w) X_{-\alpha-\beta}(w) \psi_{\alpha}(w): \\
& -(-1)^{p(\alpha) p(\beta)} \epsilon(\alpha) \frac{c_{\alpha, \beta}}{z-w}: X_{\alpha+\beta}(w) X_{-\alpha}(w) \psi^{\beta}(w): \\
& -(-1)^{p(\alpha)} \frac{(\alpha \mid \alpha)}{z-w}: X_{-\alpha}(w) \psi^{\alpha}(w) \psi_{\alpha}(w) \psi_{\alpha}(w): \\
& -(-1)^{p(\beta)} \epsilon(\alpha) \epsilon(\beta) \frac{(\alpha \mid \beta)}{z-w}: X_{-\alpha}(w) \psi_{\alpha}(w) \psi^{\beta}(w) \psi_{\beta}(w): \\
& +(-1)^{p(\alpha)(p(\beta)+1)} \frac{1}{z-w}: X_{\beta}(w) \psi^{\beta}(w) \psi^{\alpha}(w) \psi_{\alpha}(w):
\end{aligned}
$$

Looking at the above formulas, one sees that all of the extra terms cancel out and disappear if the conditions $(\alpha \mid \alpha)=(\beta \mid \beta)=0,(\alpha \mid \beta)=$ 1, and $\epsilon(\alpha)=\epsilon(\beta)=1$ and $c_{\alpha, \beta}=1$ are satisfied, and has the following:

Lemma 2.3. Assume that $(\alpha \mid \alpha)=(\beta \mid \beta)=0,(\alpha \mid \beta)=1$, and $\epsilon(\alpha)=$ $\epsilon(\beta)=1$ and $c_{\alpha, \beta}=1$. Then

1)

$$
B(z) G_{+}(w)=(K+1)\left\{\frac{G_{+}(w)}{(z-w)^{2}}+\frac{\partial G_{+}(w)}{z-w}\right\}
$$

2)

$$
B(z) G_{-}(w)=(K+1)\left\{\frac{2 G_{-}(w)}{(z-w)^{2}}+\frac{\partial G_{-}(w)}{z-w}\right\} .
$$

The other products $A(z) A(w), A(z) B(w)$ and $B(z) B(w)$ are obtained from the above Lemmas by using the Borcherds-Jacobi identity (see, e.g., the formula (4.6.7) in 《4):

$$
\left[a_{(m)}, b_{(n)}\right]=\sum_{j \geq 0}\left(\begin{array}{c}
m \\
j
\end{array}\right)\left(a_{(j)} b\right)_{(m+n-j)},
$$

for mutually local fields $a(z)=\sum_{n \in \mathbb{Z}} a_{(n)} z^{-n-1}$ and $b(z)=\sum_{n \in \mathbb{Z}} b_{(n)} z^{-n-1}$. Actually the calculation goes as follows. Put

$$
\begin{aligned}
& A(z)=\sum_{n \in \mathbb{Z}} A_{(n)} z^{-n-1}, \\
& B(z)=\sum_{n \in \mathbb{Z}} B_{(n)} z^{-n-1},
\end{aligned}
$$


and

$$
G_{ \pm}(z)=\sum_{n \in \mathbb{Z}} G_{ \pm(n)} z^{-n-1}
$$

Then the above lemmas, together with skew-symmetry, give the following:

$$
\begin{aligned}
& G_{+(n)} G_{-}= \begin{cases}B & \text { if } n=0, \\
A & \text { if } n=1, \\
-2 K & \text { if } n=2 \\
0 & \text { if } n \geq 3\end{cases} \\
& G_{-(n)} G_{+}= \begin{cases}B-\partial A & \text { if } n=0 \\
-A & \text { if } n=1 \\
-2 K & \text { if } n=2 \\
0 & \text { if } n \geq 3\end{cases} \\
& A_{(n)} G_{ \pm}=-G_{ \pm(n)} A= \begin{cases} \pm(K+1) G_{ \pm} & \text {if } n=0 \\
0 & \text { if } n \geq 1\end{cases} \\
& B_{(n)} G_{+}= \begin{cases}(K+1) \partial G_{+} & \text {if } n=0 \\
(K+1) G_{+} & \text {if } n=1 \\
0 & \text { if } n \geq 2\end{cases} \\
& G_{+(n)} B= \begin{cases}0 & \text { if } n=0 \text { or } n \geq 2, \\
(K+1) G_{+} & \text {if } n=1,\end{cases} \\
& B_{(n)} G_{-}=G_{-(n)} B= \begin{cases}(K+1) \partial G_{-} & \text {if } n=0, \\
2(K+1) G_{-} & \text {if } n=1, \\
0 & \text { if } n \geq 2 .\end{cases}
\end{aligned}
$$

So, by (2.9) applied to $a=G_{+}$and $b=G_{-}$, one has

$$
\begin{aligned}
& {\left[G_{+(0)}, G_{-(n)}\right]=B_{(n)},} \\
& {\left[G_{+(1)}, G_{-(n)}\right]=B_{(n+1)}+A_{(n)},}
\end{aligned}
$$

and, using these, can compute $A_{(n)} A, A_{(n)} B, B_{(n)} A$ and $B_{(n)} B$; e.g.,

$$
B_{(n)} A=G_{+(0)} G_{-(n)} A+G_{-(n)} G_{+(0)} A= \begin{cases}(K+1) \partial A & \text { if } n=0, \\ (K+1) A & \text { if } n=1, \\ 2 K(K+1) & \text { if } n=2, \\ 0 & \text { if } n \geq 3 .\end{cases}
$$


Translating into the terminology of operator products, one has the following:

Lemma 2.4 .

$$
\begin{aligned}
& A(z) A(w)=\frac{-4 K(K+1)}{(z-w)^{2}} \\
& A(z) B(w)=(K+1)\left\{\frac{-2 K}{(z-w)^{3}}+\frac{A(w)}{(z-w)^{2}}\right\}, \\
& B(z) A(w)=(K+1)\left\{\frac{2 K}{(z-w)^{3}}+\frac{A(w)}{(z-w)^{2}}+\frac{\partial A(w)}{z-w}\right\}, \\
& B(z) B(w)=(K+1)\left\{\frac{2 B(w)}{(z-w)^{2}}+\frac{\partial B(w)}{z-w}\right\} .
\end{aligned}
$$

Summing up the above, one obtains the following :

Theorem 2.1. Let $\left\{\alpha_{1}, \alpha_{2}\right\}$ (resp. $\left\{\alpha_{1}^{\vee}, \alpha_{2}^{\vee}\right\}$ ) be the set of simple roots (resp. simple coroots) of the Lie superalgebra sl(2|1) such that

$$
\left(\left\langle\alpha_{i}^{\vee}, \alpha_{j}\right\rangle\right)_{i, j=1,2}=\left(\left(\alpha_{i}^{\vee} \mid \alpha_{j}^{\vee}\right)\right)_{i, j=1,2}=\left(\begin{array}{ll}
0 & 1 \\
1 & 0
\end{array}\right),
$$

where $(\mid$ ) is the super-invariant super-symmetric bilinear form. Choose $X_{\alpha} \in \mathfrak{g}_{\alpha}$, for each root $\alpha$, satisfying the conditions (2.1), (2.2) and $c_{\alpha_{1}, \alpha_{2}}=1$, where $H_{\alpha_{i}}=\alpha_{i}^{\vee}$. Then the fields

$$
\begin{aligned}
G^{\prime}(z) & :=X_{\alpha_{1}}(z) \psi^{\alpha_{1}}(z)+X_{-\alpha_{2}}(z) \psi^{-\alpha_{2}}(z), \\
G^{\prime *}(z) & :=X_{-\alpha_{1}}(z) \psi^{-\alpha_{1}}(z)+X_{\alpha_{2}}(z) \psi^{\alpha_{2}}(z), \\
A(z) & :=K\left\{-: \psi^{\alpha_{1}}(z) \psi_{\alpha_{1}}(z):+: \psi^{\alpha_{2}}(z) \psi_{\alpha_{2}}(z):\right\}+\left(H_{\alpha_{1}}-H_{\alpha_{2}}\right)(z), \\
B(z) & :=K\left\{-: \partial \psi^{\alpha_{1}}(z) \psi_{\alpha_{1}}(z):+: \psi^{\alpha_{2}}(z) \partial \psi_{\alpha_{2}}(z):\right\} \\
& +\sum_{i=1,2}\left\{: X_{\alpha_{i}}(z) X_{-\alpha_{i}}(z):+: H_{\alpha_{i}}(z) \psi^{\alpha_{i}}(z) \psi_{\alpha_{i}}(z):\right\} \\
& -\partial H_{\alpha_{2}}(z)+: X_{\alpha_{1}+\alpha_{2}}(z) \psi^{\alpha_{1}}(z) \psi^{\alpha_{2}}(z):-: X_{-\alpha_{1}-\alpha_{2}}(z) \psi_{\alpha_{1}}(z) \psi_{\alpha_{2}}(z):
\end{aligned}
$$

satisfy the following product formulas:

$$
\begin{aligned}
G^{\prime}(z) G^{\prime}(w) & =G^{* *}(z) G^{* *}(w)=0, \\
G^{\prime}(z) G^{*}(w) & =\frac{-2 K}{(z-w)^{3}}+\frac{A(w)}{(z-w)^{2}}+\frac{B(w)}{z-w}, \\
A(z) G^{\prime}(w) & =\frac{K+1}{z-w} G^{\prime}(w), \\
A(z) G^{*}(w) & =\frac{-(K+1)}{z-w} G^{\prime *}(w),
\end{aligned}
$$




$$
\begin{aligned}
& B(z) G^{\prime}(w)=(K+1)\left\{\frac{G^{\prime}(w)}{(z-w)^{2}}+\frac{\partial G^{\prime}(w)}{z-w}\right\} \\
& B(z) G^{*}(w)=(K+1)\left\{\frac{2 G^{* *}(w)}{(z-w)^{2}}+\frac{\partial G^{\prime *}(w)}{z-w}\right\} \\
& A(z) A(w)=\frac{-4 K(K+1)}{(z-w)^{2}}, \\
& A(z) B(w)=(K+1)\left\{\frac{-2 K}{(z-w)^{3}}+\frac{A(w)}{(z-w)^{2}}\right\} \\
& B(z) A(w)=(K+1)\left\{\frac{2 K}{(z-w)^{3}}+\frac{A(w)}{(z-w)^{2}}+\frac{\partial A(w)}{z-w}\right\} \\
& B(z) B(w)=(K+1)\left\{\frac{2 B(w)}{(z-w)^{2}}+\frac{\partial B(w)}{z-w}\right\}
\end{aligned}
$$

Putting

$$
\begin{aligned}
L(z) & :=\frac{1}{K+1}\left\{B(z)-\frac{1}{2} \partial A(z)\right\}, & & J(z):=\frac{1}{K+1} A(z), \\
G(z) & :=\frac{1}{K+1} G^{\prime}(z), & & G^{*}(z):=G^{*}(z), \\
K^{\prime} & :=\frac{-2 K}{K+1}, & &
\end{aligned}
$$

one can rewrite the above into the the standard product formulas of the $\mathrm{N}=2$ superconformal algebra as follows:

\section{Corollary 2.1 .}

$$
\begin{aligned}
L(z) L(w) & =\frac{\frac{1}{2} K^{\prime}}{(z-w)^{4}}+\frac{2 L(w)}{(z-w)^{2}}+\frac{\partial L(w)}{z-w} \\
L(z) J(w) & =\frac{J(w)}{(z-w)^{2}}+\frac{\partial J(w)}{z-w}, \quad J(z) L(w)=\frac{J(w)}{(z-w)^{2}} \\
L(z) G(w) & =\frac{\frac{3}{2} G(w)}{(z-w)^{2}}+\frac{\partial G(w)}{z-w}, \quad G(z) L(w)=\frac{\frac{3}{2} G(w)}{(z-w)^{2}}+\frac{\frac{1}{2} \partial G(w)}{z-w}, \quad G^{*}(z) L(w)=\frac{\frac{3}{2} G^{*}(w)}{(z-w)^{2}}+\frac{\frac{1}{2} \partial G^{*}(w)}{z-w}, \\
L(z) G^{*}(w) & =\frac{\frac{3}{2} G^{*}(w)}{(z-w)^{2}}+\frac{\partial G^{*}(w)}{z-w}, \\
J(z) J(w) & =\frac{2 K^{\prime}}{(z-w)^{2}}, \\
J(z) G(w) & =\frac{G(w)}{z-w}, \quad G(z) J(w)=\frac{-G(w)}{z-w}
\end{aligned}
$$




$$
\begin{aligned}
J(z) G^{*}(w) & =\frac{-G^{*}(w)}{z-w}, \quad G^{*}(z) J(w)=\frac{G^{*}(w)}{z-w} \\
G(z) G^{*}(w) & =\frac{K^{\prime}}{(z-w)^{3}}+\frac{J(w)}{(z-w)^{2}}+\frac{L(w)+\frac{1}{2} \partial J(w)}{z-w} \\
G^{*}(z) G(w) & =\frac{K^{\prime}}{(z-w)^{3}}-\frac{J(w)}{(z-w)^{2}}+\frac{L(w)-\frac{1}{2} \partial J(w)}{z-w} \\
G(z) G(w) & =G^{*}(z) G^{*}(w)=0 .
\end{aligned}
$$

We note that this superconformal algebra $\mathfrak{A}$ has important commutants. The following theorem is easily shown from (1.5) and (1.6b):

Theorem 2.2. Introduce two fields:

$$
d_{1}(z):=X_{\alpha_{1}+\alpha_{2}}(z)+: \psi_{\alpha_{1}}(z) \psi_{\alpha_{2}}(z):
$$

and

$$
d_{2}(z):=X_{-\alpha_{1}-\alpha_{2}}(z)-: \psi^{\alpha_{1}}(z) \psi^{\alpha_{2}}(z): .
$$

Then,

$$
d_{i}(z) G(w)=d_{i}(z) G^{*}(w)=0 \text { for } i=1,2 .
$$

This implies, by the Borcherds-Jacobi identity (2.9), that

$$
\left[d_{i(m)}, G_{(n)}\right]=\left[d_{i(m)}, G_{(n)}^{*}\right]=0
$$

for all $m, n \in \mathbb{Z}$ and $i=1,2$.

Let $V$ be the tensor product of a highest weight $\widehat{s l}(2 \mid 1)$-module and the symmetric algebra over $\left\{\psi^{\alpha_{i}}(n) ; i=1,2\right.$ and $\left.n<0\right\}$. Then $V$ is an $\mathfrak{A}$-module and, by (2.10), all simultaneous eigenspaces of $d_{i(m)}$, $i=1,2$ and $m \in \mathbb{Z}$, are stable under the action of $G_{(n)}$ and $G_{(n)}^{*}$, and so are $\mathfrak{A}$-submodules.

3. The Centerless $N=4$ superconformal algebra via $\widehat{A}(1,1)$

Let us consider the superalgebra $\operatorname{sl}(2 \mid 2)$ with simple roots $\Pi=$ $\left\{\alpha_{1}, \alpha_{2}, \alpha_{3}\right\}$ and Chevalley generators $\left(e_{i}, f_{i}, \alpha_{i}^{\vee}\right)_{i=1,2,3}$, satisfying

$$
\left(\left\langle\alpha_{i}^{\vee}, \alpha_{j}\right\rangle\right)_{i, j=1,2,3}=\left(\begin{array}{ccc}
0 & 1 & 0 \\
1 & -2 & 1 \\
0 & 1 & 0
\end{array}\right) .
$$


The algebra $\operatorname{sl}(2 \mid 2)$ has a super-invariant super-symmetric bilinear form ( $\mid$ ) such that

$$
\left(\left(\alpha_{i}^{\vee} \mid \alpha_{j}^{\vee}\right)\right)_{i, j=1,2,3}=\left(\begin{array}{ccc}
0 & 1 & 0 \\
1 & -2 & 1 \\
0 & 1 & 0
\end{array}\right) .
$$

From this, one sees that $s l(2 \mid 2)$ is not simple, since the Cartan matrix (3.1) is singular of rank 2 , or in other words, the inner product defined by (3.2) is degenerate, and that $\alpha_{1}^{\vee}-\alpha_{3}^{\vee}$ spans the center. The quotient simple superalgebra $s l(2 \mid 2) / \mathbb{C} \cdot\left(\alpha_{1}^{\vee}-\alpha_{3}^{\vee}\right)$ is called $A(1,1)$. Its Cartan subalgebra $\mathfrak{h}$ is, therefore, 2-dimensional, and each $\alpha_{i}$ naturally defines a linear form on $\mathfrak{h}$, which is also denoted by $\alpha_{i}$ using the same characters. Under this notation, the induced inner product on the dual space $\mathfrak{h}^{*}$ satisfies

$$
\left(\left(\alpha_{i} \mid \alpha_{j}\right)\right)_{i, j=1,2,3}=\left(\begin{array}{ccc}
0 & 1 & 0 \\
1 & -2 & 1 \\
0 & 1 & 0
\end{array}\right) .
$$

For simplicity, we write $\left(m_{1}, m_{2}, m_{3}\right)$ in place of $\sum_{i=1}^{3} m_{i} \alpha_{i}$, and also $-\left(m_{1}, m_{2}, m_{3}\right)$ in place of $-\sum_{i=1}^{3} m_{i} \alpha_{i}$. Then the sets $\Delta^{+}, \Delta_{\text {even }}^{+}$and $\Delta_{\text {odd }}^{+}$of roots of $A(1,1)$ are given as follows:

$$
\begin{aligned}
\Delta_{\text {even }}^{+} & =\{(0,1,0), \quad(1,1,1)\}, \\
\Delta_{\text {odd }}^{+} & =\{(1,0,0), \quad(0,0,1),(1,1,0), \quad(0,1,1)\}, \\
\Delta^{+} & =\Delta_{\text {even }}^{+} \cup \Delta_{\text {odd }}^{+} .
\end{aligned}
$$

We note that the square length of a root $\alpha$ is equal to -2 if $\alpha= \pm \alpha_{2}$, 2 if $\alpha= \pm(1,1,1)$, and 0 if $\alpha$ is odd. For each root $\alpha=\left(m_{1}, m_{2}, m_{3}\right)$ we choose a root vecter $X_{\alpha}=X_{\left(m_{1}, m_{2}, m_{3}\right)}$ in such a way that

$$
\begin{aligned}
& e_{(1,1,0)}:=\left[e_{1}, e_{2}\right], e_{(0,1,1)}:=\left[e_{2}, e_{3}\right], \quad e_{(1,1,1)}:=\left[e_{1}, e_{(0,1,1)}\right]=\left[e_{(1,1,0)}, e_{3}\right], \\
& f_{(1,1,0)}:=\left[f_{1}, f_{2}\right], \quad f_{(0,1,1)}:=\left[f_{2}, f_{3}\right], \quad f_{(1,1,1)}:=\left[f_{1}, f_{(0,1,1)}\right]=\left[f_{(1,1,0)}, f_{3}\right] .
\end{aligned}
$$

We now consider the affinization $\widehat{A}(1,1)$, and the following fields:

$$
\begin{aligned}
& G_{1}(z):=X_{(1,1,0)}(z) \psi^{\alpha_{1}}(z)-X_{-(0,0,1)}(z) \psi^{-\alpha_{3}}(z), \\
& G_{1}^{*}(z):=-X_{-(1,1,0)}(z) \psi^{-\alpha_{1}}(z)+X_{(0,0,1)}(z) \psi^{\alpha_{3}}(z), \\
& G_{2}(z):=X_{(1,0,0)}(z) \psi^{\alpha_{1}}(z)-X_{-(0,1,1)}(z) \psi^{-\alpha_{3}}(z), \\
& G_{2}^{*}(z):=-X_{-(1,0,0)}(z) \psi^{-\alpha_{1}}(z)+X_{(0,1,1)}(z) \psi^{\alpha_{3}}(z), \\
& A_{1}(z):=K\left\{: \psi^{\alpha_{3}}(z) \psi_{\alpha_{3}}(z):-: \psi^{\alpha_{1}}(z) \psi_{\alpha_{1}}(z):\right\}-\alpha_{2}^{\vee}(z), \\
& A_{2}(z):=K\left\{: \psi^{\alpha_{3}}(z) \psi_{\alpha_{3}}(z):-: \psi^{\alpha_{1}}(z) \psi_{\alpha_{1}}(z):\right\}+\alpha_{2}^{\vee}(z), \\
& B_{1}(z):=K\left\{: \psi^{\alpha_{3}}(z) \partial \psi_{\alpha_{3}}(z):-: \partial \psi^{\alpha_{1}}(z) \psi_{\alpha_{1}}(z):\right\}
\end{aligned}
$$




$$
\begin{aligned}
& -: X_{(1,1,0)}(z) X_{-(1,1,0)}(z):+: X_{(0,0,1)}(z) X_{-(0,0,1)}(z): \\
& -:\left(\alpha_{1}^{\vee}+\alpha_{2}^{\vee}\right)(z) \psi^{\alpha_{1}}(z) \psi_{\alpha_{1}}(z):-: \alpha_{3}^{\vee}(z) \psi^{\alpha_{3}}(z) \psi_{\alpha_{3}}(z): \\
& +\partial \alpha_{3}^{\vee}(z)+: X_{(1,1,1)}(z) \psi^{\alpha_{1}}(z) \psi^{\alpha_{3}}(z):+: X_{-(1,1,1)}(z) \psi_{\alpha_{1}}(z) \psi_{\alpha_{3}}(z):, \\
B_{2}(z) & :=K\left\{: \psi^{\alpha_{3}}(z) \partial \psi_{\alpha_{3}}(z):-: \partial \psi^{\alpha_{1}}(z) \psi_{\alpha_{1}}(z):\right\} \\
& -: X_{(1,0,0)}(z) X_{-(1,0,0)}(z):-: X_{(0,1,1)}(z) X_{-(0,1,1)}(z): \\
& -:\left(\alpha_{2}^{\vee}+\alpha_{3}^{\vee}\right)(z) \psi^{\alpha_{3}}(z) \psi_{\alpha_{3}}(z):-: \alpha_{1}^{\vee}(z) \psi^{\alpha_{1}}(z) \psi_{\alpha_{1}}(z): \\
& +\partial\left(\alpha_{2}^{\vee}+\alpha_{3}^{\vee}\right)(z)+: X_{(1,1,1)}(z) \psi^{\alpha_{1}}(z) \psi^{\alpha_{3}}(z): \\
& +: X_{-(1,1,1)}(z) \psi_{\alpha_{1}}(z) \psi_{\alpha_{3}}(z):
\end{aligned}
$$

Then Theorem 2.1 applied to each quadruplet $\left(G_{i}, G_{i}^{*}, A_{i}, B_{i}\right), i=$ 1,2 , gives the following:

$$
\begin{gathered}
G_{i}(z) G_{i}(w)=G_{i}^{*}(z) G_{i}^{*}(w)=0 \\
G_{i}(z) G_{i}^{*}(w)=\frac{-2 K}{(z-w)^{3}}+\frac{A_{i}(w)}{(z-w)^{2}}+\frac{B_{i}(w)}{z-w}, \\
A_{i}(z) G_{i}(w)=\frac{K+1}{z-w} G_{i}(w), \\
A_{i}(z) G_{i}^{*}(w)=\frac{-(K+1)}{z-w} G_{i}^{*}(w) \\
B_{i}(z) G_{i}(w)=(K+1)\left\{\frac{G_{i}(w)}{(z-w)^{2}}+\frac{\partial G_{i}(w)}{z-w}\right\}, \\
B_{i}(z) G_{i}^{*}(w)=(K+1)\left\{\frac{2 G_{i}^{*}(w)}{(z-w)^{2}}+\frac{\partial G_{i}^{*}(w)}{z-w}\right\} .
\end{gathered}
$$

One also has the following:

$$
\begin{gathered}
e_{2}(z) G_{1}(w)=0, \quad e_{2}(z) G_{2}(w)=\frac{-G_{1}(w)}{z-w}, \\
e_{2}(z) G_{1}^{*}(w)=\frac{G_{2}^{*}(w)}{z-w}, \quad e_{2}(z) G_{2}^{*}(w)=0, \\
f_{2}(z) G_{1}(w)=\frac{G_{2}(w)}{z-w}, \quad f_{2}(z) G_{2}(w)=0, \\
f_{2}(z) G_{1}^{*}(w)=0, \quad f_{2}(z) G_{2}^{*}(w)=\frac{-G_{1}^{*}(w)}{z-w} .
\end{gathered}
$$




$$
\begin{array}{ll}
\alpha_{2}^{\vee}(z) G_{1}(w)=\frac{-G_{1}(w)}{z-w}, & \alpha_{2}^{\vee}(z) G_{2}(w)=\frac{G_{2}(w)}{z-w} . \\
\alpha_{2}^{\vee}(z) G_{1}^{*}(w)=\frac{G_{1}^{*}(w)}{z-w}, & \alpha_{2}^{\vee}(z) G_{2}^{*}(w)=\frac{-G_{2}^{*}(w)}{z-w},
\end{array}
$$

and

$$
\begin{array}{ll}
A_{1}(z) G_{2}(w)=\frac{K-1}{z-w} G_{2}(w), & A_{1}(z) G_{2}^{*}(w)=\frac{1-K}{z-w} G_{2}^{*}(w), \\
A_{2}(z) G_{1}(w)=\frac{K-1}{z-w} G_{1}(w), & A_{2}(z) G_{1}^{*}(w)=\frac{1-K}{z-w} G_{1}^{*}(w) .
\end{array}
$$

The operator products $G_{i}(z) G_{j}^{*}(w)$ for $i \neq j$ are obtained from Lemma 1.1 as follows:

$$
\begin{gathered}
G_{1}(z) G_{2}(w)=G_{1}^{*}(z) G_{2}^{*}(w)=0, \\
G_{1}(z) G_{2}^{*}(w)=-\frac{2 e_{2}(w)}{(z-w)^{2}}-\frac{\partial e_{2}(w)}{z-w} \\
+\frac{1}{z-w}\left\{e_{2}(w)\left(: \psi^{\alpha_{3}}(w) \psi_{\alpha_{3}}(w):-: \psi^{\alpha_{1}}(w) \psi_{\alpha_{1}}\left(w_{(3)}: 1\right) \mathrm{b}\right)\right. \\
\left.-: X_{(1,1,0)}(w) X_{-(1,0,0)}(w):-: X_{(0,1,1)}(w) X_{-(0,0,1)}(w):\right\}, \\
G_{2}(z) G_{1}^{*}(w)=\frac{2 f_{2}(w)}{(z-w)^{2}}+\frac{\partial f_{2}(w)}{z-w} \\
+\frac{1}{z-w}\left\{f _ { 2 } ( w ) \left(: \psi^{\alpha_{1}}(w) \psi_{\alpha_{1}}(w):-: \psi^{\alpha_{3}}(w) \psi_{\alpha_{3}}\left(w_{(3)}: 11 \mathrm{c}\right)\right.\right. \\
\left.-: X_{(1,0,0)}(w) X_{-(1,1,0)}(w):-: X_{(0,0,1)}(w) X_{-(0,1,1)}(w):\right\} .
\end{gathered}
$$

We now introduce the field

$$
\begin{aligned}
D(z) & :=B_{1}(z)-B_{2}(z)+\partial \alpha_{2}^{\vee}(z) \\
& =: X_{(1,0,0)}(z) X_{-(1,0,0)}(z):+: X_{(0,1,1)}(z) X_{-(0,1,1)}(z): \\
& -: X_{(1,1,0)}(z) X_{-(1,1,0)}(z):-: X_{(0,0,1)}(z) X_{-(0,0,1)}(z): \\
& +\alpha_{2}^{\vee}(z)\left(: \psi^{\alpha_{3}}(z) \psi_{\alpha_{3}}(z):-: \psi^{\alpha_{1}}(z) \psi_{\alpha_{1}}(z):\right) .
\end{aligned}
$$

Then one has

$$
\begin{aligned}
D(z) e_{2}(w) & =\frac{2}{z-w}\left\{: X_{(1,1,0)}(w) X_{-(1,0,0)}(w):+: X_{(0,1,1)}(w) X_{-(0,0,1)}(w):\right. \\
& \left.+e_{2}(w)\left(: \psi^{\alpha_{1}}(w) \psi_{\alpha_{1}}(w):-: \psi^{\alpha_{3}}(w) \psi_{\alpha_{3}}(w):\right)\right\},
\end{aligned}
$$




$$
\begin{aligned}
D(z) f_{2}(w) & =\frac{2}{z-w}\left\{: X_{(1,0,0)}(w) X_{-(1,1,0)}(w):+: X_{(0,0,1)}(w) X_{-(0,1,1)}(w):\right. \\
& \left.+f_{2}(w)\left(: \psi^{\alpha_{3}}(w) \psi_{\alpha_{3}}(w):-: \psi^{\alpha_{1}}(w) \psi_{\alpha_{1}}(w):\right)\right\} .
\end{aligned}
$$

Let $\tilde{\mathfrak{A}}$ be the vertex algebra generated by $G_{i}, G_{i}^{*}(i=1,2), e_{2}, f_{2}$, $\alpha_{2}^{\vee}$, and $\mathfrak{I}$ its ideal generated by $D$, and we consider the vertex algebra $\mathfrak{A}:=\tilde{\mathfrak{A}} / \mathfrak{I}$. Then, by $(3.13 \mathrm{a})$ and $(3.13 \mathrm{~b})$, the third terms in the righthand sides of (3.11b) and (3.11c) vanish in $\mathfrak{A}$, and one has

$$
\begin{gathered}
G_{1}(z) G_{2}^{*}(w)=-\left\{\frac{2 e_{2}(w)}{(z-w)^{2}}+\frac{\partial e_{2}(w)}{z-w}\right\}, \\
G_{2}(z) G_{1}^{*}(w)=\frac{2 f_{2}(w)}{(z-w)^{2}}+\frac{\partial f_{2}(w)}{z-w} .
\end{gathered}
$$

As a cost of this ideal $\mathfrak{I}$, we have to assume henceforward that $K=$ 0 , since otherwise $G_{i}$ and $G_{i}^{*}$ belong to $\mathfrak{I}$ and the vertex algebra $\mathfrak{A}$ collapses. Then

$$
A_{1}=-A_{2}=-\alpha_{2}^{\vee}
$$

and all other products are computed from $(3.4) \sim\left(3.11^{\prime}\right)$ by the BorcherdsJacobi identity (2.9) in a similar way as is explained in Section 2. And by putting

$$
\begin{aligned}
L(z) & :=B_{1}(z)-\frac{1}{2} \partial A_{1}(z)=B_{2}(z)-\frac{1}{2} \partial A_{2}(z), \\
h(z) & :=-\alpha_{2}^{\vee}(z), \quad e(z):=-e_{2}(z), \quad f(z):=f_{2}(z),
\end{aligned}
$$

one sees that these fields satisfy the product formulas of the $N=4$ superconformal algebra:

Theorem 3.1.

$$
\begin{aligned}
& L(z) L(w)=\frac{2 L(w)}{(z-w)^{2}}+\frac{\partial L(w)}{z-w}, \\
& L(z) X(w)=\frac{X(w)}{(z-w)^{2}}+\frac{\partial X(w)}{z-w}, \quad X(z) L(w)=\frac{X(w)}{(z-w)^{2}}, \\
& X(z) Y(w)=\frac{[X, Y](w)}{z-w} \quad \text { for } X, Y=h, \text { e, or } f \\
& L(z) F(w)=\frac{\frac{3}{2} F(w)}{(z-w)^{2}}+\frac{\partial F(w)}{z-w} \\
& F(z) L(w)=\frac{\frac{3}{2} F(w)}{(z-w)^{2}}+\frac{\frac{1}{2} \partial F(w)}{z-w} \quad \text { for } F=G_{i}, G_{i}^{*}, \\
& h(z) G_{1}(w)=-G_{1}(z) h(w)=\frac{G_{1}(w)}{z-w}, \quad h(z) G_{1}^{*}(w)=-G_{1}^{*}(z) h(w)=\frac{-G_{1}^{*}(w)}{z-w}
\end{aligned}
$$




$$
\begin{aligned}
h(z) G_{2}(w) & =-G_{2}(z) h(w)=\frac{-G_{2}(w)}{z-w}, \quad h(z) G_{2}^{*}(w)=-G_{2}^{*}(z) h(w)=\frac{G_{2}^{*}(w)}{z-w} \\
e(z) G_{1}(w) & =G_{1}(z) e(w)=0, \quad e(z) G_{1}^{*}(w)=-G_{1}^{*}(z) e(w)=\frac{-G_{2}^{*}(w)}{z-w}, \\
e(z) G_{2}(w) & =-G_{2}(z) e(w)=\frac{G_{1}(w)}{z-w}, \quad e(z) G_{2}^{*}(w)=G_{2}^{*}(z) e(w)=0, \\
f(z) G_{1}(w) & =-G_{1}(z) f(w)=\frac{G_{2}(w)}{z-w}, \quad f(z) G_{1}^{*}(w)=G_{1}^{*}(z) f(w)=0, \\
f(z) G_{2}(w) & =G_{2}(z) f(w)=0, \quad f(z) G_{2}^{*}(w)=-G_{2}^{*}(z) f(w)=\frac{-G_{1}^{*}(w)}{z-w}, \\
G_{i}(z) G_{j}(w) & =G_{i}^{*}(z) G_{j}^{*}(w)=0, \quad \frac{h(w)}{(z-w)^{2}}+\frac{L(w)+\frac{1}{2} \partial h(w)}{z-w} \\
G_{1}(z) G_{1}^{*}(w) & =G_{2}^{*}(z) G_{2}(w)=\frac{-h(w)}{(z-w)^{2}}+\frac{L(w)-\frac{1}{2} \partial h(w)}{z-w} \\
G_{2}(z) G_{2}^{*}(w) & =G_{1}^{*}(z) G_{1}(w)=\frac{2 e(w)}{(z-w)^{2}}+\frac{\partial e(w)}{z-w} \\
G_{1}(z) G_{2}^{*}(w) & =-G_{2}^{*}(z) G_{1}(w)=\frac{2 f(w)}{(z-w)^{2}}+\frac{\partial f(w)}{z-w} \\
G_{2}(z) G_{1}^{*}(w) & =-G_{1}^{*}(z) G_{2}(w)=\frac{2(w)}{(z)}
\end{aligned}
$$

Commutants for $\mathfrak{A}$ are given as follows in this case:

Theorem 3.2. Consider two fields:

$$
d_{1}(z):=X_{(1,1,1)}(z)+: \psi_{\alpha_{1}}(z) \psi_{\alpha_{3}}(z):
$$

and

$$
d_{2}(z):=X_{-(1,1,1)}(z)+: \psi^{\alpha_{1}}(z) \psi^{\alpha_{3}}(z):
$$

Then

$$
d_{i}(z) G_{j}(w)=d_{i}(z) G_{j}^{*}(w)=0 \text { and } \quad d_{i}(z) X(w)=0,
$$

for $i, j=1,2$ and $X=h, e, f$.

From this theorem, one has

$$
\left[d_{i(m)}, G_{j(n)}\right]=\left[d_{i(m)}, G_{j(n)}^{*}\right]=0
$$

and

$$
\left[d_{i(m)}, X_{(n)}\right]=0
$$

for $i, j=1,2, X=h, e, f$, and $m, n \in \mathbb{Z}$. Then, by (3.12), all $d_{i(m)}$ 's commute with $D_{(n)}$ and so, when we consider representations, commute with the action of the vertex algebra $\mathfrak{A}$. And all simultaneous eigenspaces of $d_{i(m)}, i=1,2$ and $m \in \mathbb{Z}$, are $\mathfrak{A}$-submodules. 


\section{REFERENCES}

[1] S. Hosono and A. Tsuchiya : Lie algebra cohomology and N=2 SCFT based on the GKO construction, Commun. Math. Phys. 136 (1991), 451-486.

[2] V. G. Kac : Lie superalgebras, Adv. in Math. 26 (1977), 8-96.

[3] V. G. Kac : Infinite dimensional Lie algebras, third edition, Cambridge University Press, 1990.

[4] V. G. Kac : Vertex Algebras for Beginners, University Lecture Series Vol. 10, American Mathematical Society, 1996.

[5] V. G. Kac: Superconformal algebras and transitive group actions on quadrics, preprint, 1996. 